\title{
Intraspecific genetic variation matters when predicting seagrass distribution under climate change
}

\author{
Zi-Min $\mathrm{Hu}^{1}$, Quan-Sheng Zhang ${ }^{1}$, Jie Zhang ${ }^{2}$, Jamie Kass ${ }^{3}$, Mammola Stefano ${ }^{4}$, Pablo \\ Fresia $^{5}$, Draisma Stefano ${ }^{6}$, Jorge Assis ${ }^{7}$, Alexander Jueterbock ${ }^{8}$, Masashi Yokota ${ }^{9}$, and \\ Zhixin Zhang ${ }^{9}$ \\ ${ }^{1}$ Yantai University \\ ${ }^{2}$ Chinese Academy of Sciences \\ ${ }^{3}$ Okinawa Institute of Science and Technology Graduate University \\ ${ }^{4}$ University of Helsinki \\ ${ }^{5}$ Institut Pasteur de Montevideo \\ ${ }^{6}$ Prince of Songkla University \\ ${ }^{7}$ University of Algarve \\ ${ }^{8}$ Nord University \\ ${ }^{9}$ Tokyo University of Marine Science and Technology
}

March 4, 2021

\begin{abstract}
Seagrasses play a vital role in structuring coastal marine ecosystems, but their distributional range and genetic diversity have declined rapidly over the past decades. In order to improve conservation of seagrass species, it is important to predict how climate change may impact their ranges. Such predictions are typically made with correlative species distribution models (SDMs), which can estimate a species' potential distribution under present and future climatic scenarios given species' presence data and climatic predictor variables. However, these models are typically constructed with species-level data, and thus ignore intraspecific genetic variability of populations that potentially have adaptations to heterogeneous climatic conditions. Here, we explore the link between intraspecific adaptation and niche differentiation in Thalassia hemprichii, a seagrass broadly distributed in the tropical Indo-Pacific Ocean and a crucial provider of habitat for numerous marine species. Using microsatellite-based genotyping, we identified two distinct phylogeographical lineages within the nominal species and found an intermediate level of differentiation in their multidimensional environmental niches, suggesting the possibility for local adaptation. We then compared projections of the species' habitat suitability under climate change scenarios using species-level and lineage-level SDMs. In the Central Tropical Indo-Pacific region, both models predicted considerable range contraction in the future, but the lineage-level model predicted more severe habitat loss. The two modelling approaches predicted opposite pattern in habitat change in the Western Tropical Indo-Pacific region. Our results highlight the necessity of conserving distinct populations and genetic pools under climate change and have important implications for guiding future management of seagrasses.
\end{abstract}

\section{Introduction}

Marine ecosystems worldwide are experiencing dramatic shifts in environmental conditions due to climate change, the most evident of which is a steady increase in sea surface temperature (SST) (Cheunget al. 2013). These changes can affect marine organisms in different ways, such as by altering the structure of trophic webs (e.g., Hyndes et al. 2016), biasing sex ratios in species with temperature-dependent sex determination 
(e.g., Miyoshi et al.2020), and redefining the geographical ranges of species (e.g., Pinskyet al. 2020). In order to guide natural resource management under this changing marine landscape, it is crucial to make future predictions of suitable habitat for target species as accurately as possible.

Species distribution models (SDMs), which estimate relationships between species' presence data and environmental predictors, have been used extensively to predict potential changes in species' distributions under climate change scenarios (Guisan et al. 2017). The majority of SDMs are constructed at the species-level or even higher taxonomic levels, and this is particularly true for applications to marine species (Robinsonet al. 2011; Robinson et al. 2017; Chefaoui et al.2018; Jayathilake \& Costello 2018; Melo-Merino et al. 2020). One fundamental and critical assumption underlying species-level SDMs is niche conservatism, which assumes that all populations of a species have analogous environmental requirements and respond in a similar way to a changing environment (Guisan et al. 2017; Smith et al.2019). But this assumption ignores intra-specific variation, in particular local adaptation and phenotypic plasticity, which are frequently observed especially in broadly distributed taxa (e.g., Marín-Guirao et al. 2016; Duarte et al. 2018; King et al.2018; Benito Garzón et al. 2019; Peterson et al. 2019; Zhang et al. 2020b).

SDMs constructed with data for lineages below the species level can account for possible local adaptations and therefore can provide more reliable niche estimations and habitat suitability projections for species with intraspecific variation. For instance, a species-level SDM for the threatened Japanese crayfish Cambaroides japonicus (De Haan 1841) predicted that this species might lose a large proportion of its suitable habitat in the future, whereas lineage-level SDMs for the same species predicted a weaker impact of climate change overall (Zhanget al. 2021). The importance of taxonomic units (i.e., above and below the species level) in distribution modelling has recently been recognized (Benito Garzón et al. 2019; Peterson et al.2019; Smith et al. 2019; Collart et al. 2021), which has resulted in more SDM applications for terrestrial and freshwater species that consider intra-specific variation (e.g., Ikeda et al. 2017; Razgour et al. 2019; Zhang et al. 2021). Conversely, relatively few SDM studies have investigated this issue in the marine realm (but see Assis et al. 2018a; Cacciapaglia \& van Woesik 2018; Lowen et al. 2019).

Seagrasses are one of the most critical habitat engineers (along with seaweeds, mangroves, and coral reefs) of tropical coastal marine environments. They not only harbor rich marine biodiversity in seagrass meadows, but also provide a number of ecosystem services, such as primary productivity, habitat restoration, resources for marine life, and human recreation, among others (Unsworth et al. 2018). Maintaining these services is key to achieving conservation and economic goals under global change. Yet, seagrass ecosystems are declining worldwide at an annual rate of $7 \%$ due to multiple natural and human-mediated disturbances (Orth et al. 2006; Waycott et al. 2009). It is noteworthy that climate change has received considerable attention as a major factor for the increasing loss of seagrass meadows (Jordà et al. 2012; Thomson et al. 2015; Repolho et al. 2017; Duarte et al. 2018; Smale et al. 2019). This is particularly true for the tropical Indo-Pacific bioregion, which supports the most seagrass diversity and a high diversity of associated flora and fauna (Short et al. 2007) but has suffered from striking degradation of seagrass coverage (Coleset al. 2011; Rasheed \& Unsworth 2011; Grech et al. 2012; Chefaoui et al. 2018; Olsen et al. 2018; Brodie et al. 2020). Given the global ecological roles of seagrasses, it is crucial to make accurate forecasts of their distribution patterns in the face of climate change, but seagrasses are "among the least-studied groups" (Melo-Merino et al. 2020) with respect to range shift projections. The majority (if not all) of SDM studies on seagrasses have been at the species level and therefore did not incorporate potential intraspecific variation.

The seagrass Thalassia hemprichii (Ehrenberg) Ascherson (Hydrocharitaceae) is a perennial climax species that is widely distributed in the tropical Indo-Pacific bioregion (Green \& Short 2003), extending from Australia, the peripheral limit of its eastern range (Hernawan et al. 2017), to East Africa in the West Indian Ocean (Jahnke et al. 2019a). It reproduces sexually via seeds and asexually via vegetative growth of rhizomes. Uprooted adult plants can potentially float for months and hence colonize distant areas (Wuet al. 2016). In addition, this seagrass forms buoyant seeds that remain afloat for long enough to disperse a few hundreds of kilometers (Lacap et al. 2002). A recent survey revealed that seedlings can also disperse for over a month due to the accumulation of oxygen in the body tissue (Wu et al. 2016). Thus, T. hemprichii has excellent long- 
distance dispersal potential that may play a significant role in shaping population genetic structure (Lowe $\&$ Allendorf 2010). This species may be particularly vulnerable to climate change because it exhibits spatial separation of the sexes (dioecious), reinforced by physiological and morphological differentiation of each sex to variable microhabitats (Hultine et al. 2016). Recent genetic studies of T. hemprichii detected genetic lineage divisions in the East and West Indo-Pacific Ocean (Hernawan et al. 2017; Jahnke et al. 2019a), but we still do not have a clear understanding of the distribution of lineages across the entire tropical Indo-Pacific region, or whether these diverged lineages are expected to respond differentially to climate change.

In the present study, we used T. hemprichii as a model to: (i) examine range-wide divergence of genetic lineages in the tropical Indo-Pacific Ocean; (ii) test if phylogeographical lineages exist, and if so, quantify niche differentiation between distinct lineages; (iii) predict climate change impacts on the species' range with species-level and lineage-level SDMs. By incorporating potential intra-specific variation, our SDMs can provide more realistic predictions on how climate change will shift future distributions of a habitat-forming seagrass, thus generating valuable knowledge for guiding the long-term management of this species in the tropical Indo-Pacific coast.

\section{Materials and methods}

\section{Datasets and intraspecific genetic clustering}

We sampled the microsatellite datasets of two recently published regional studies, i.e., 17 populations in the East Indo-Pacific (Hernawan et al. 2017) and 11 populations in the Western Indian Ocean (Jahnke et al. 2019a). We used twelve microsatellites (i.e., Thh3, Thh15, Thh34, Thh41, TH07, TH34, TH37, TH43, TH52, TH66, TH73) for population structuring and lineage sorting of 1021 individuals from 28 populations across the tropical Indo-Pacific (Fig. 1a). We then estimated pairwise genetic differences among populations using the Cavalli-Sforza and Edwards chord distance and represented them in a network using the R package IGRAPH (Csardi \& Nepusz 2006) with the addition of a custom script by Johansson et al. (2015). To visually inspect the relationships within and between the main genetic clusters inferred by STRUCTURE (Pritchard et al. 2000), we pruned the full network by sequentially removing edges (i.e., network pairwise links among sampling sites) of decreasing genetic distance until the point at which the main groups of tightly connected nodes still remained connected (in order to avoid the split of any large network cluster from the main network). We estimated the classification of sampling sites within network communities at each step of the pruning process with the "fastgreedy" community detection algorithm implemented in IGRAPH (Clauset et al. 2004, Blondel et al. 2008). Network analysis (Fig. 1b), Bayesian-based STRUCTURE (Fig. 1c), and molecular variation (AMOVA) (Table S1 in Supporting Information) revealed strong overall genetic differentiation among two distinct lineages occupying the Tropical Indo-Pacific. Based on the landscape genetic analysis of Cushman et al. (2014) and the definitions of global marine ecoregions (Spalding et al. 2007), we classified these two lineages as distinct genotypes encompassed within two biogeographic regions: the Western Tropical Indo-Pacific (WTIP) and the Central Tropical Indo-Pacific (CTIP). We then used the two lineages in subsequent ecological niche modelling.

\section{Distribution data and marine predictors}

We collected a total of 62,465 presence records of $T$. hemprichiifrom a recently assembled and cleaned dataset of global marine forests (Assis et al. 2020) and published literature (see Data availability). In SDM studies, it is critical to correct for sampling bias and remove clustered records, which may over-represent environmental conditions in better-surveyed regions (Kramer-Schadtet al. 2013). Therefore, presence records were filtered by: i) removing duplicated records at the resolution of our environmental predictors (i.e., keeping only one record per 5 arcmin grid cell); ii) removing records on land or with distance to land $>370 \mathrm{~km}$ (following other SDM studies for coastal species; e.g., Zhang et al. 2020a), and iii) performing spatial thinning using a distance of $20 \mathrm{~km}$ using the $\mathrm{R}$ package $s p$ Thin (Aiello-Lammens et al.2015). This distance is a reasonable 
approximation of the dispersal potential for this plant traveling via floating propagules (Lacapet al. 2002), and it can also reduce potential effects of sampling bias while retaining sufficient numbers of presence records for our analyses. As significant clustering was present in the data (particularly around Australia), these procedures removed a good proportion of the presence data. Ultimately, we kept 519 records for the species-level model (hereafter "species model"), 479 records for the CTIP lineage-level model (hereafter "CTIP model"), and 26 records for the WTIP lineage-level model (hereafter "WTIP model") (Fig. 1a).

It is important to properly select the extent of the study area used to sample background records when constructing presence-background SDMs for target species (Barve et al. 2011; Vale et al. 2014). Given previous marine SDMs (e.g., Zhang et al. 2020a) and the geographical range of T. hemprichii, we restricted our study to the areas within $370 \mathrm{~km}$ of land between $25^{\circ} \mathrm{E}$ and $180^{\circ} \mathrm{E}$, and between $50^{\circ} \mathrm{S}$ and $40^{\circ} \mathrm{N}$ (Fig. 1a). Please note that our study extent includes southern Australia and New Zealand, where this species does not naturally occur. It is always challenging to estimate an appropriate study extent for a species (Barve et al. 2011), but the extent we selected should represent the plausible accessible areas to T. hemprichii over evolutionary time. We subsetted this main study extent to create separate study extents for the WTIP and CTIP lineages (Fig. 1a) based on our molecular results (see details in the Lineage genetic diversity in the Results section).

A number of marine predictors have been demonstrated to influence the geographical distribution of marine species (Bosch et al. 2018). Based on previous studies (e.g., Jayathilake \& Costello 2018; Zhanget al. 2020a), we initially considered twenty such predictors for modeling, including two geographical predictors (water depth and distance to land) from the Global Marine Environment Datasets (http://gmed.auckland.ac.nz; Basher et al. 2018) and eighteen environmental predictors (including annual mean, maximum, minimum, range, average of the minimum records per year, and average of the maximum records per year) for SST, sea surface salinity, and sea surface current velocity from the Bio-ORACLE database v2.1 (https://www.biooracle.org; Assis et al. 2018b). In SDM studies, highly collinear predictors can lead to spurious interpretations of variable importance and unexpected predictions if correlations change in different projection scenarios (Dormann et al. 2013). Hence, we checked collinearity by calculating the pairwise Pearson's correlation coefficients $(r)$ among the twenty predictors (Supporting Information Fig. S1) and selected one among highly correlated predictors $(|r|>0.7)$ (Dormann et al.2013) based on present-day and future data availability, biological importance, and previous findings on important variables for estimating seagrass distribution (Jayathilake \& Costello 2018). In the end, we retained the two geographical predictors and six environmental predictors: annual mean current velocity, minimum current velocity, annual mean sea surface salinity, annual range of sea surface salinity, annual mean SST, and annual range of SST.

To project future habitat suitability of $T$. hemprichii, we considered four representative concentration pathway (RCP) scenarios (i.e., RCP 2.6, RCP 4.5, RCP 6.0, and RCP 8.5), and two time periods (i.e., 2050s: the average for 2040-2050s; and 2100s: the average for 2090-2100). We obtained the corresponding projections of future marine environmental layers from the Bio-ORACLE database v2.1. We assumed that the two geographical predictors would remain unchanged for future projections (Zhang et al. 2020a).

\section{Niche differentiation estimation}

To estimate whether the two lineages of $T$. hemprichii occupy different niche spaces, we characterized their realized niches using Hutchinsonian $n$-dimensional hypervolumes (Hutchinson 1957) sensu Blonder et al.(2018). We quantified the realized niches of the WTIP and CTIP lineages using the eight selected marine predictor variables (see previous section). In short, we extracted and standardized (i.e., zero means and unit variance) marine predictor values associated with the presence records for the two lineages. We then determined the volumes and shapes of the realized niches with the $\mathrm{R}$ package hypervolume using the Gaussian method (Blonder 2019). We measured the extent of niche differentiation between the two lineages with the kernel.betafunction (Mammola \& Cardoso 2020) in the R package BAT (Cardosoet al. 2015, 2020). Following Carvalho \& Cardoso (2020), niche differentiation between hypervolumes was partitioned into the following two processes: niche shift (replacement of space between hypervolumes) and niche contraction/expansion (net 
difference between hypervolumes). The niche differentiation index ranges from 0 (niches overlap entirely) to 1 (niches are fully dissimilar) (Carvalho \& Cardoso 2020; Mammola \& Cardoso 2020).

\section{Species distribution modelling}

We built SDMs using Maxent 3.4.4, a presence-background machine learning algorithm with two main complexity tuning parameters: regularization multiplier, which penalizes complexity by removing predictors with low predictive ability, and feature class, which allows for increasing complexity of the model response (Phillipset al. 2017). For each model (species model, WTIP model, and CTIP model), we randomly generated 10,000 background points within the corresponding study region. As Maxent's default settings for the main tuning parameters can result in overfit models (Radosavljevic \& Anderson 2014), we used a version of the R package ENMeval under expansion (1.9.0; https://github.com/jamiemkass/ENMeval) to tune our Maxent models over ranges of each parameter and chose models with optimal complexity based on performance metrics calculated on withheld data (Muscarella et al. 2014). In brief, we considered a total of 32 candidate models with different combinations of regularization multipliers (RM; ranging from 0.5 to 4.0 , at 0.5 interval), which penalize complexity more with higher values, and feature classes (linear, quadratic, hinge), which allow responses with differing flexibility. Rather than using conventional random cross-validation to judge model performance, we used a spatial block cross-validation approach, which typically results in evaluations that better reflect the model's ability to transfer to non-analog conditions (Roberts et al. 2017; Valavi et al. 2019). Briefly, each study region was divided into four spatial blocks containing an equal number of presence records, three blocks were used for model training and the remaining block for validation, then this procedure was repeated until every block was used for model validation. As with previous studies (e.g., Radosavljevic \& Anderson 2014; Kass et al. 2020), the optimal model was selected by sequentially considering a $10 \%$ omission rate (i.e., the percentage of validation presences with habitat suitability predictions lower than that of the 10th quantile of training predictions), followed by the area under the receiver operating characteristics curve (AUC) calculated on the validation data (i.e., the model's ability to discriminate between presence and background records) to break ties. We acknowledge that AUC is a poor measure for the absolute performance of presence-background models (e.g., Jiménez-Valverde 2012), but nonetheless this metric can be used to make relative comparisons of candidate models fitted with the same data (Loboet al. 2008).

Predictive performances of the three best-performing Maxent models were further assessed using the continuous Boyce index, a reliable evaluation measure of presence-only algorithms (Hirzel et al. 2006). The continuous Boyce index ranges from -1 to 1 , where positive values suggest that model predictions match well with the presence data, and negative values suggest a poor match (Hirzel et al. 2006). Variable importance for each model was determined using permutation importance calculated by Maxent. For this method, presence and background data values for each predictor variable in turn were randomly permuted and training AUC recalculated - a large drop in AUC indicates higher importance (Phillips 2017). In addition, we estimated the marginal response curves of important predictors (i.e., curves representing habitat suitability along a range of the values of one predictor variable while keeping the other predictors constant). We converted continuous habitat suitability predictions for $T$. hemprichii to binary values using the same $10 \%$ omission thresholds that we used for model evaluation (Radosavljevic \& Anderson 2014). We then transformed the binary habitat suitability projections to the Lambert Cylindrical Equal Area projection at a resolution of $10 \mathrm{~km}$ and calculated areas of potential distribution (Zhang et al. 2020a).

It is of great importance to consider species dispersal ability into SDMs when estimating climate change impacts (Araujo et al. 2006; Guisan et al. 2017). Given the relatively high dispersal ability of T. hemprichii (Lacap et al. 2002) and a lack of apparent dispersal barriers in marine environments (Robinson et al. 2011), we estimated range size change under an unlimited dispersal scenario, which assumes that species have unrestricted dispersal ability and can disperse to any suitable area (Araujo et al. 2006; Zhanget al. 2020c). Range size change was calculated as follows:

range size change $=\frac{\text { future suitable area }- \text { present suitable area }}{\text { present suitable area }} \times 100 \%$, 
where negative and positive values represent range contraction and expansion, respectively.

We used the optimal species- and lineage-level models to make projections of future potential distribution based on the different RCP scenarios for the two future time periods. Making projections using SDMs into novel environmental space (i.e., outside the range of training data) results in some degree of extrapolations, which should be quantified to determine levels of uncertainty (Elith et al.2010). Therefore, we measured the similarity between present-day and future environmental conditions using multivariate environmental similarity surfaces (MESS) (Elith et al. 2010). In practice, we calculated the MESS with the R package rmaxent (Baumgartner \& Wilson 2021) for each model using the top three most important predictors via permutation importance: positive MESS values indicate conditions more similar to the training data, while negative values indicate conditions more different (i.e., novel).

\section{Results}

\section{Lineage genetic diversity}

We found significant genetic divergence between the populations of T. hemprichii in the Western Tropical Indo-Pacific and Central Tropical Indo-Pacific regions. Although we found some minor discrepancies (see Data availability) between the two datasets after carefully inspecting the calibrated fragment lengths of the microsatellites (Hernawan et al. 2017; Jahnke et al.2019a), even after deleting a few microsatellites (e.g., Thh41, TH07 and TH37), two genetic lineages in T. hemprichii remained significantly diverged (i.e., CTIP and WTIP) across the Tropical Indo-Pacific (Fig. 1b, 1c). Genetic variation among lineages accounted for $43.42 \%$ of the total genetic variation $\left(\Phi_{\mathrm{CT}}=0.43, P<0.0001\right.$; Supporting Information Table S1). Very limited genetic admixture was observed between the CTIP and WTIP lineages. The CTIP lineage harbored strikingly rich genetic diversity, with three times more alleles and allelic richness, and eight times fewer private alleles than the WTIP lineage (Supporting Information Table S2).

\section{Niche differentiation between hypervolumes}

The size of the realized niche of the CTIP lineage was one order of magnitude greater than that of the WTIP lineage (CTIP lineage: 17295.6; WTIP lineage: 2273.2) (Fig. 2). Niche differentiation between the two hypervolumes (0.97) was mainly due to variation in niche size (0.79), whereas niche shift contributed only marginally (0.18). Difference in realized niches was easily distinguished via water depth and distance to land, with the WTIP lineage selecting a narrow range of water depth and distance to land (Fig. 2). The two lineages also exhibited niche differentiation with respect to annual mean sea surface salinity. In addition, the CTIP lineage niche was broader with respect to annual mean SST and annual range SST, whereas that of the WTIP lineage was broader for annual mean current velocity, minimum current velocity, and annual range of sea surface salinity (Fig. 2). Niche differentiation between the two hypervolumes was also high (0.86) when we considered only marine environmental predictors (i.e., excluding water depth and distance to land) (Supporting Information Fig. S2).

\section{Model performance}

The tuning parameter settings with optimal complexity for the species-level and lineage-level models ranged from relatively simple to complex. The optimal species-level model was the most complex (hinge features and 0.5 RM), while those for the lineage-level models were simpler (CTIP: linear/quadratic/hinge features and 2.5 RM; WTIP: linear/quadratic features and $0.5 \mathrm{RM}$ ) (Table 1). The average $10 \%$ omission rate was considerably lower for the WTIP lineage-level model (3.57\%) than for the other models (CTIP: 26.69\%; species: 17.93\%; Table 1) - as this was lower than the expection of average $10 \%$ omission for the metric, it indicates that the optimal settings results in models that may over-predict to some extent for WTIP. Although omission rate was used primarily for model selection, the average validation AUC scores used to 
break ties were very high for all optimal models (Table 1); we think this is due to the fact that a majority of presence data are in near-shore waters (Fig. 1a), which likely inflated the model's ability to discriminate between these presences and background records in deeper water. In addition, all three optimal models had relatively high continuous Boyce index scores (over 0.90; Table 1), indicating that final model predictions matched the presence data well. The eight predictors had different levels of importance in the three models, but water depth and distance to land consistently played important roles (Table 2). In particular, these two predictors accounted for more than $95 \%$ of permutation importance in the WTIP model (Table 2). For the CTIP and species models, annual mean SST also had a high permutation importance $(\sim 29 \%$ and $\sim 24 \%$, respectively) (Table 2). Response curves for water depth and distance to land suggest that shallow coastal waters are more suitable for T. hemprichii (Supporting Information Fig. S3).

\section{Present-day habitat suitability projections}

Under present-day conditions, species and lineage models projected similar but not identical habitat suitability patterns, with a large part of the East African coast and the Pacific region as suitable habitat for this species (Fig. 3). Compared with the species model, the CTIP model predicted more southern distribution in Australia (Fig. 3c, 3d). In particular, the CTIP model predicted suitable conditions in the Spencer Gulf, Southern Australia, where the species does not naturally occur (Fig. 3a, 3c). The species model did not capture this pattern (Fig. 3b, 3d). Moreover, the WTIP model identified more suitable habitat in the Red Sea than the species-level model (Fig. 3c, 3d). Overall, species- and lineage-level models predicted comparable suitable areas for T. hemprichii in the WTIP region (species model: 302,800 square km; WTIP model: 315,000 square $\mathrm{km}$ ), while the species model predicted broader suitable area for the CTIP region (species model: 1,873,800 square km; CTIP model: 1,757,900 square km).

\section{Climate change impacts on habitat suitability}

Species- and lineage-level models resulted in different future habitat suitability projections in the CTIP region, with the lineage-level model resulting in predictions of more loss of suitable areas (Table 3; Fig. 4). Both species- and lineage-level models predict considerable future loss of suitable area in the CTIP region, especially on the Sunda Shelf (i.e., Indonesia and Malaysia) (Table 3; Fig. 4). Compared with the species model, the CTIP model projected more extensive range loss under all climatic scenarios (Table 3). Interestingly, both models predicted that the species will shift slightly southwards in Australia.

Species-level and lineage-level models predicted different impacts of climate change on habitat suitability for T. hemprichii in the WTIP region (Table 3). The WTIP model predicted range expansion (except under the RCP 2.6 scenario for the 2050s), whereas the species model consistently indicated range contraction (Table 3). Overall, both species- and lineage-level models predicted that future climate change marginally affects habitat suitability in the WTIP region and that changes in range size were mostly $<15 \%$, with the exception of a higher value $(\sim 24 \%)$ for the species model in the 2100 s for the RCP 8.5 scenario (Table 3 ). The WTIP model predicted that habitat suitability of T. hemprichii in the WTIP region will remain stable in the future, while the species model predicted range contraction in the Red Sea and expansion in southern Madagascar and South Africa (Fig. 4).

Both species and CTIP models consistently showed that MESS values in the Sunda Shelf were slightly negative, which demonstrates small differences in climatic conditions between the present-day and future scenarios for this region (Supporting Information Fig. S4). For the WTIP region, the lineage and species model showed high environmental similarity except slight environmental dissimilarity in the Red Sea between present-day and future scenarios (Supporting Information Fig. S4). These results indicate a low degree of extrapolation in our model predictions. 


\section{Discussion}

This study identified two diverged genetic lineages (WTIP and CTIP) in the seagrass T. hemprichii across the tropical Indo-Pacific. The observed niche differentiation between the two lineages suggests a violation of the niche conservatism assumption for species-level SDMs, and our lineage-level predictions of present and future range importantly avoid this assumption. Despite differences between the habitat suitability predictions of the lineage-level and species-level SDMs, they consistently predict that the CTIP lineage is at greater risk of range contraction in the future. For this seagrass, but also for other taxa with intraspecific genetic differentiation, incorporating information about phylogeographical structure when modelling the impacts of climate change provides more realistic predictions to better understand future range shifts (Smith et al. 2019; Zhang et al. 2021).

\section{Critical marine predictor variables for seagrasses}

Both the lineage-level and species-level SDMs showed that distance to land, water depth, and annual mean SST represent the most essential factors in explaining the distributional patterns of T. hemprichii . The predominant roles of the two geographical predictors and the negligible roles of marine environmental predictors in the WTIP lineage-level model (Table 2) may partially explain the marginal impacts of climate change predicted for this region. The importance of these three predictors has been emphasized in previous studies of Thalassia species (e.g., Duarte 1991; Lapointe et al.1994; Fourqurean \& Zieman 2002; Zhang et al. 2014) and other seagrasses (e.g., Baumstark et al. 2016; Jayathilake \& Costello 2018). For instance, Jayathilake \& Costello (2018) used a set of 13 predictors and developed SDMs for 60 seagrass species including T. hemprichii . They reported the important roles of distance to land and mean SST in explaining geographical distributions of seagrasses. Unexpectedly, maximum SST was reported to be critical, but water depth was less important in their study (Jayathilake \& Costello 2018). This inconsistency in our study might be attributed to (a) different sets of predictors, and/or (b) different roles of marine predictors in different seagrass species.

\section{Incorporating intraspecific variation into SDMs for seagrasses}

Seagrasses provide vital ecological services in marine ecosystems and SDMs have been applied to this taxonomic group for multiple purposes (see reviews by Robinson et al. 2011; Robinson et al.2017; MeloMerino et al. 2020). Nonetheless, all previously reported SDMs on seagrasses were built at the species level and thus have not considered possible intraspecific variation. For instance, Chefaoui et al. (2018) developed species-level SDMs for two seagrasses (Posidonia oceanica and Cymodocea nodosa) in the Mediterranean Sea and predicted that the two species are likely to experience dramatic habitat loss in the future. We fully agree that species-level SDMs are by definition informative; but given the high prevalence of intraspecific variation in marine macrophytes (e.g., Kinget al. 2018), and the significance of intraspecific variation in SDMs (Benito Garzón et al. 2019; Smith et al. 2019; Zhanget al. 2021; Collart et al. 2021), incorporating intraspecific genetic variation into forecasts of seagrass distribution should result in more realistic scenarios of the potential consequences of climate change.

The importance of taxonomic resolution in SDMs has been addressed in several terrestrial and freshwater species, but much more rarely for marine species (see review by Smith et al. 2019; Collart et al. 2021). Specieslevel SDMs that disregard existing intraspecific variation can either over- or under-estimate climate change impact on distributional change. For instance, species-level models for the lodgepole pine Pinus contorta consistently predicted more extreme habitat loss than subspecies-level models, regardless of the dispersal scenario (i.e., no or unlimited dispersal ability) (Oney et al.2013). As another example, although a specieslevel model for the reef-building coral Porites lobata predicted over $5 \%$ habitat expansion, when modelling this species as five genetically isolated subpopulations the prediction was ca. $50 \%$ habitat loss (Cacciapaglia \& van Woesik 2018). In the present study, the species model consistently predicted low impacts of climate change in the CTIP region in comparison to the lineage model (e.g., the habitat loss vs. stability in the Sunda 
Shelf in Fig. 4c vs. Fig. 4d). As for the WTIP region, we found the opposite pattern. Here, the lineage model predicted stable future habitats in the southern Red Sea (Fig. 4c), whereas the species model predicted habitat loss, including to the north of Mauritius (Fig. 4d). In addition, both species and lineage models predict a southward range expansion in the southern CTIP, but only the species model clearly predicts this in the WTIP. Southern expansion is likely correlated with future temperature increases in areas which are now too cold (Supporting Information Fig. S5a). We should note that MESS values in the equatorial regions were slightly negative, which indicates novel future environmental conditions. This is due in part to higher future SST values for this region than those used by the present-day SDM (Supporting Information Fig. S5b) - thus, SDM projections in this region should be associated with more uncertainty. Further studies involving both field investigations and associated data updates and methodological developments for models [e.g., developing ensembles of small models (Breiner et al. 2018) or using smaller study extent] would further improve our predictions for climate change impacts on T. hemprichii in the Tropical Indo-Pacific.

\section{Intraspecific variation and local adaptation in seagrass}

Differences in response to thermal changes related to intraspecific variation, whether eco-physiological or evolutionary, are well-documented in seagrasses (King et al. 2018). This variation, partly based on phenotypic plasticity or local adaptation, ultimately might permit seagrasses to acclimatize and adapt to changes in climate (Duarte et al. 2018). The marine predictor variables that played a predominant role in our SDMs (e.g., annual mean SST and water depth) could be responsible for both long- and short-term local adaptation of $T$. hemprichii to a changing climate (King et al. 2018; Jahnke et al. 2019b). In support of this, common-garden experiments have revealed a clear local adaptation to increased temperatures in Zostera marina (Franssen et al. 2011; 2014), and to a depth gradient in Posidonia oceanica(Marín-Guirao et al. 2016; Jahnke et al. 2019b). Further, parallel adaptation of $Z$. marina to thermal clines along the American and European coasts was demonstrated using a space-for-time substitution design and gene expression profiling (Jueterbock et al. 2016). Such adaptive local differentiation induced by divergent environmental forces (e.g., light, depth and temperature) has led to structured populations and lineages in seagrasses at various spatial scales (Dattolo et al. 2014; Jueterbock et al. 2016; Jahnke et al. 2019b), suggesting that adaptation to local conditions is a key mechanism for seagrasses to face global climate change.

In T. hemprichii, natural selection imposed by environmental heterogeneity might have resulted in the evolution of locally adapted populations with considerable variation in productivity, growth rate and competitive interactions (Martins \& Bandeira 2001; Lyimo et al.2006; Larkum et al. 2018). Despite clear genetic differentiation identified between the WTIP and CTIP lineages, we did not ascertain the adaptive and nonadaptive components of divergence in a common landscape of the tropical Indo-Pacific. Future studies should focus on distinguishing neutral genetic differentiation from local adaptation using reciprocal transplant trials (e.g., common gardens and provenance trials) (see Joyce \& Rehfeldt 2013; Ralph et al. 2018). Also, it is most important to assess the sub-lethal susceptibility of T. hemprichii to thermal stress before the strongest impacts of future climate change are sustained. Intraspecific genetic diversity across populations can increase a species' adaptive capacity and result in cascading effects to the entire ecosystem (Evans et al. 2017). It is thus important to identify the most temperature-tolerant genotypes from the WTIP and CTIP lineages, perhaps by manipulating temperature to quantify the performance of individual genotypes of $T$. hemprichiiacross thermal gradients. It is also essential to clarify whether genotype complementarity or dominance enhance the adaptive capacity in a population (Hughes \& Stachowicz 2011).

\section{Conservation implications}

The challenge of designing effective actions for seagrass conservation in the Indo-Pacific exists in the gap between science, policy, and practice (Fortes 2018). In this study, the separation in geographic distribution and high niche differentiation between the CTIP and WTIP lineages suggest that T. hemprichii populations may be locally adapted (Merilä \& Hendry 2014). For species with significant intraspecific genetic diversity, it is crucial to help maintain the species' potential for adaptive responses to climate change by conserving this 
diversity (D'Amen et al. 2013). In particular, lineage differentiation can be explained by recruitment rate (Lyimoet al. 2006; Sherman et al. 2018), nutrient resorption (Martins \& Bandeira 2001), and evolutionary history from the origin center to the distributional margins (Mukai 1993). Dramatic future habitat loss in the CTIP was predicted by both the species- and lineage-level models (Fig. 4), stressing the urgency to develop monitoring programs to rescue evolutionary and/or ecologically important units in T. hemprichii , particularly the populations and gene pools that have persisted through past long-term climate change because of local adaptation (Bell 2017; Hernawan et al. 2017). Furthermore, the recognition of high niche differentiation between the WTIP and CTIP lineages may help to establish coherent principles and regulating practices by which the different areas that $T$. hemprichii inhabits can be protected efficiently.

The biomass, abundance, and productivity of seagrasses are highly correlated with both habitat suitability (Martins \& Bandeira 2001; Saunders et al. 2013) and epiphytic species biodiversity (Lyimoet al. 2008). Optimizing productivity of $T$. hemprichii in a given site or population can help to increase associated community diversity (Eklöf et al. 2006; Lyimo et al. 2008). Thus, it is necessary to explore how community diversity and structure correlate with the genetic composition and structure of the foundational species $T$. hemprichii. Such research can help validate the results of SDMs in this study and quantify the relationship between T. hemprichii and its relevant community components (Ikeda et al.2017). Since populations in each of the CTIP and WTIP lineages are locally adapted, policymakers and stakeholders are encouraged to use local seed sources of T. hemprichii to ensure management strategies for successful restoration and conservation purposes. However, as the WTIP lineage may be more resilient to future climate change, WTIP seeds could possibly be used to restore CTIP seagrass beds which are predicted to disappear in the future.

Finally, apart from marine geographical and environmental predictors, geographical distributions of seagrasses are also determined by other factors including biotic interactions. For instance, Hyndes et al. (2016) predicted that accelerating tropicalization can lead to a potential shift both among the seagrass themselves and among their associated communities, thereby affecting ecosystem services that seagrasses provide in this region. The importance of incorporating biotic interactions into SDMs has long been recognized but it is still poorly addressed in the marine realm. More mechanistic studies underlying thermal adaptation by linking ecology to genetics should be done to better understand how T. hemprichii will adapt to climate change (Duarte et al. 2018; Hu et al. 2020).

\section{Acknowledgements}

We thank Xiaokai Fan (Ocean University of China, China) for collecting distribution data of Thalassia hemprichii and Jinxin Zhou (University of Tokyo, Japan) for figure illustration. We further thank Dr Aussanee Meekaew (Shizuoka University, Japan) for kindly providing us with the nice pictures of Thalassia hemprichii in Figure 2. This study was supported by National Natural Science Foundation of China (31971395, 41761144057), Thailand Research Fund (RDG6130002), the Foundation for Science and Technology (FCT) through the projects UID/Multi/04326/2019 and PTDC/BIA-CBI/6515/2020 and the transitional norm -DL57/2016/CP1361/CT0035 to JA. SM acknowledges support from the European commission (program H2020-MSCA-IF-2019; grant award: 882221). ZZ and MY acknowledge the financial support from the Japan Society for the Promotion of Science Grant-in-Aid for Scientific Research (C) (18K05780). JMK acknowledges support from the Japan Society for the Promotion of Science Postdoctoral Fellowships for Foreign Researchers program and the Okinawa Institute of Science and Technology Graduate University.

\section{References}

Aiello-Lammens, M. E., Boria, R. A., Radosavljevic, A., Vilela, B., \& Anderson, R. P. (2015). spThin: an $\mathrm{R}$ package for spatial thinning of species occurrence records for use in ecological niche models.Ecography, $38,541-545$. 
Araújo, M. B., Thuiller, W., \& Pearson, R. G. (2006). Climate warming and the decline of amphibians and reptiles in Europe. Journal of Biogeography , 33, 1712-1728.

Assis, J., Fragkopoulou, E., Frade, D., Neiva, J., Oliveira, A., Abecasis, D., .. \& Serrão, E. A. (2020). A fine-tuned global distribution dataset of marine forests. Scientific Data, 7, 119.

Assis, J., Serrão, E. A., C. Coelho, N., Tempera, F., Valero, M., \& Alberto, F. (2018a). Past climate changes and strong oceanographic barriers structured low - latitude genetic relics for the golden kelpLaminaria ochroleuca . Journal of Biogeography, 45, 2326-2336.

Assis, J., Tyberghein, L., Bosch, S., Verbruggen, H., Serrao, E. A., \& De Clerck, O. (2018b). Bio-ORACLE v2.0: Extending marine data layers for bioclimatic modelling. Global Ecology and Biogeography , 27, 277-284.

Barve, N., Barve, V., Jimenez-Valverde, A., Lira-Noriega, A., Maher, S. P., Peterson, A. T., .. \& \& Villalobos, F. (2011). The crucial role of the accessible area in ecological niche modeling and species distribution modeling. Ecological Modelling , 222, 1810-1819.

Basher, Z., Bowden, D. A., \& Costello, M. J. (2018). Global Marine Environment Datasets (GMED). World Wide Web electronic publication. Version 2.0 (Rev.02.2018). Accessed at http://gmed.auckland.ac.nz

Baumgartner, J., \& Wilson, P. (2021). rmaxent : Tools for working with Maxent in R. R package version 0.8.5.9000. https://github.com/johnbaums/rmaxent

Baumstark, R., Duffey, R., \& Pu, R. L. (2016) Mapping seagrass and colonized hard bottom in Springs Coast, Florida using WorldView-2 satellite imagery. Estuarine, Coastal and Shelf Science, 181, 83-92.

Bell, G. (2017). Evolutionary rescue. Annual Review of Ecology, Evolution and Systematics , 48, 605-627.

Benito Garzon, M., Robson, T. M., \& Hampe, A. (2019). $\Delta$ Trait SDMs: species distribution models that account for local adaptation and phenotypic plasticity. New Phytologist , 222, 1757-1765.

Blondel, V. D., Guillaume, J. L., Lambiotte, R., \& Lefebvre, E. (2008). Fast unfolding of communities in large networks. Journal of Statistical Mechanics, P10008.

Blonder, B., Morrow, C. B., Maitner, B., Harris, D. J., Lamanna, C., Violle, C., .. \& Kerkhoff, A. J. (2018). New approaches for delineating $n$-dimensional hypervolumes. Methods in Ecology and Evolution, 9, 305-319.

Blonder, B., with contributions from Harris, D. J. (2019). Hypervolume: High Dimensional Geometry and Set Operations Using Kernel Density Estimation, Support Vector Machines, and Convex Hulls. R package version 2.0.12. https://CRAN.R-project.org/package=hypervolume

Bosch, S., Tyberghein, L., Deneudt, K., Hernandez, F., \& De Clerck, O. (2018). In search of relevant predictors for marine species distribution modelling using the MarineSPEED benchmark dataset. Diversity and Distributions , 24, 144-157.

Breiner, F. T., Nobis, M. P., Bergamini, A., \& Guisan, A. (2018). Optimizing ensembles of small models for predicting the distribution of species with few occurrences. Methods in Ecology and Evolution , 9, 802-808.

Brodie, G., Holland, E., N'Yeurt, A. D. R., Soapi, K., \& Hills, J. (2020). Seagrasses and seagrass habitats in Pacific small island developing states: Potential loss of benefits via human disturbance and climate change. Marine Pollution Bulletin, 160, 111573.

Cacciapaglia, C., \& van Woesik, R. (2018). Marine species distribution modelling and the effects of genetic isolation under climate change. Journal of Biogeography , 45, 154-163.

Carvalho, J. C., \& Cardoso, P. (2020). Decomposing the causes for niche differentiation between species using hypervolumes. Frontiers in Ecology and Evolution , 8, 243. 
Cardoso, P., Mammola, S., Rigal, F., \& Carvalho, J. C. (2020). BAT: Biodiversity Assessment Tools. R package version 2.0.1. https://CRAN.R-project.org/package=BAT

Cardoso, P., Rigal, F., \& Carvalho, J. C. (2015). BAT-Biodiversity Assessment Tools, an R package for the measurement and estimation of alpha and beta taxon, phylogenetic and functional diversity.Methods in Ecology and Evolution , 6, 232-236.

Chefaoui, R. M., Duarte, C. M., \& Serrão, E. A. (2018). Dramatic loss of seagrass habitat under projected climate change in the Mediterranean Sea. Global Change Biology, 24, 4919-4928.

Cheung, W. W. L., Watson, R., \& Pauly, D. (2013) Signature of ocean warming in global fisheries catch. Nature , 497, 365-368.

Clauset, A., Newman, M., \& Moore, C. (2004). Finding community structure in very large networks. Physical Review E , 70, 066111.

Coles, R., Grech, A., Rasheed, M., McKenzie, L., Unsworth, R., \& Short, F. (2011). Seagrass ecology and threats in the tropical Indo-Pacific bioregion. In: Roberts SP (ed) Seagrass: Ecology, Uses and Threats. Nova Science Publishers, pp 225-239.

Csardi, G., \& Nepusz, T. (2006). The IGRAPH software package for complex network research. InterJournal Complex Systems , 1695.

Collart, F., Hedenäs, L., Broennimann, O., Guisan, A., \& Vanderpoorten, A. (2021). Intraspecific differentiation: Implications for niche and distribution modelling. Journal of Biogeography , 48, 415-426.

Cushman, S. A., Max, T., Meneses, N., Evans, L. M., Ferrier, S., Honchak, B., .. \& Allan, G. J. (2014) Landscape genetic connectivity in a riparian foundation tree is jointly driven by climatic gradients and river networks. Ecological Applications , 24, 1000-1014.

D'Amen, M., Zimmermann, N. E., \& Pearman, P. B. (2013). Conservation of phylogeographic lineages under climate change. Global Ecology and Biogeography , 22, 93-104.

Dattolo, E., Ruocco, M., Brunet, C., Lorenti, M., Lauritano, C., D’Esposito, D., DeLuca, P., Sanges, R., Mazzuca, S., \& Procaccini, G. (2014). Response of the seagrass Posidonia oceanica to different light environments: Insights from a combined molecular and photo-physiological study. Marine Environmental Research , 101, 225-236.

Dormann, C. F., Elith, J., Bacher, S., Buchmann, C., Carl, G., Carré, G., .. Lautenbach, S. (2013). Collinearity: a review of methods to deal with it and a simulation study evaluating their performance. Ecography , 36, 27-46.

Duarte, C. M. (1991). Seagrass depth limits. Aquatic Botany , 40, 363-377.

Duarte, B., Martins, I., Rosa, R., Matos, A. R., Roleda, M. Y., Reusch, T. B. H., .. \& Jueterbock, A. (2018). Climate change impacts on seagrass meadows and macroalgal forests: an integrative perspective on acclimation and adaptation potential. Frontiers in Marine Sciences , 5, 190.

Elith, J., Kearney, M., \& Phillips, S. (2010). The art of modelling range-shifting species. Methods in Ecology and Evolution , 1, 330-342.

Eklof, J. S., Henriksson, R., \& Kautsky, N. (2006) Effects of tropical open-water seaweed farming on seagrass ecosystem structure and function. Marine Ecology Progress Series , 325, 73-84.

Evans, S. M., Verges, A., \& Poore, A. G. B. (2017) Genotypic diversity and short-term response to shading stress in a threatened seagrass: does low diversity mean low resilience? Frontiers in Plant Science, 8, 1417.

Fourqurean, J. W., \& Zieman, J. C. (2002) Nutrient content of the seagrass Thalassia testudinum reveals regional patterns of relative availability of nitrogen and phosphorus in the Florida Keys USA. Biogeochemistry , 61, 229-245. 
Fortes, M. D. (2018), Seagrass ecosystem conservation in Southeast Asia needs to link science to policy and practice. Ocean and Coastal Management, 159, 51-56.

Franssen, S. U., Gu, J., Bergmann, N., Winters, G., Klostermeier, U. C., Rosenstiel, P., Bornberg-Bauer, E., \& Reusch, T. B. H. (2011). Transcriptomic resilience to global warming in the seagrassZostera marina, a marine foundation species. Proceedings of the National Academy of Sciences USA , 108, 19276-19281.

Franssen, S. U., Gu, J., Winters, G., Huylmans, A. K., Wienpahl, I., Sparwel, M., Coyer, J. A., Olsen, J. L., Reusch, T. B. H., Bornberg-Bauer, E. (2014). Genome-wide transcriptomic responses of the seagrasses Zostera marina and Nanozostera noltii under a simulated heatwave confirm functional types. Marine Genomics , 15, 65-73.

Grech, A., Chartrand-Miller, K., Erftemeijer, P., Fonseca, M., McKenzie, L, Rasheed, M., .. \& Coles, R. (2012). A comparison of threats, vulnerabilities and management approaches in global seagrass bioregions.Environmental Research Letters , 7, 024006.

Green, E. E. P., \& Short, F. T. (2003). World atlas of seagrasses. Berkeley, CA: University of California Press.

Guisan, A., Thuiller, W., \& Zimmermann, N. E. (2017). Habitat Suitability and Distribution Models: With Applications in R. Cambridge University Press, Cambridge.

Hao, T., Elith, J., Lahoz-Monfort, J. J., \& Guillera-Arroita, G. (2020). Testing whether ensemble modelling is advantageous for maximising predictive performance of species distribution models.Ecography , 43, 549558.

Hernawan, U., van Dijk K., Kendrick, G., Feng, M., Biffin, E., .. \& McMahon, K. (2017). Historical processes and contemporary ocean currents drive genetic structure in the seagrass Thalassia hemprichii in the Indo-Australian Archipelago. Molecular Ecology , 26, 1008-1021.

Hirzel, A. H., Le Lay, G., Helfer, V., Randin, C., \& Guisan, A. (2006). Evaluating the ability of habitat suitability models to predict species presences. Ecological modelling , 199, 142-152.

Hughes, A. R., \& Stachowicz, J. J. (2011). Seagrass genotypic diversity increases disturbance response via complementarity and dominance. Journal of Ecology , 99, 445-453.

Hultine, K. R., Grady, K. C., Wood, T. E., Shuster, S. M., Stella, J. C., Whitham, T. G. (2016). Climate change perils for dioecious plant species. Nature Plants , 2, 16109.

Hutchinson, G. (1957). Concluding remarks. Cold Spring Harbor Symposia on Quantitative Biology , 22, $415-427$.

Hyndes, G. A., Heck, K. L., Verges, A., Harvey, E. S., Kendrick, G. A., Lavery, P. S., .. \& Wilson, S. (2016) Accelerating tropicalization and the transformation of temperate seagrass meadows.BioScience , 66, 938-948.

Ikeda, D. H., Max, T. L., Allan, G. J., Lau, M. K., Shuster, S. M., \& Whitham, T. G. (2017). Genetically informed ecological niche models improve climate change predictions. Global Change Biology , 23, 164-176.

Jahnke, M., Gullstrom, M., Larsson, J., Asplund, M. E., Mgeleka, S., Silas, M. O., .. \& Nordlund, L. M. (2019a). Population genetic structure and connectivity of the seagrass Thalassia hemprichiiin the Western Indian Ocean is influenced by predominant ocean currents.Ecology and Evolution , 9, 8953-8964.

Jahnke, M., D'Esposito, D., Orru, L., Lamontanara, A., Dattolo, E., Badalamenti, F., Mazzuca, S., Procaccini, G., \& Orsini, L. (2019b). Adaptive responses along a depth and a latitudinal gradient in the endemic seagrass Posidonia oceanica. Heredity, 122, 233-243.

Jayathilake, D. R., \& Costello, M. J. (2018). A modelled global distribution of the seagrass biome. Biological Conservation, 226, 120-126. 
Jimenez-Valverde, A. (2012). Insights into the area under the receiver operating characteristic curve (AUC) as a discrimination measure in species distribution modelling. Global Ecology and Biogeography , 21, 498-507.

Johansson, M. L., Alberto, F., Reed, D. C., Raimondi, P. T., Coelho, N. C., Young, M. A., .. \& Serrao, E. A. (2015). Seascape drivers ofMacrocystis pyrifera population genetic structure in the northeast Pacific. Molecular Ecology , 24, 4866-4885.

Jorda, G., Marba, N., \& Duarte, C. M. (2012). Mediterranean seagrass vulnerable to regional climate warming. Nature Climate Change, 2, 821-824.

Jueterbock, A., Franssen, S. U., Bergmann, N., Gu, J., Coyer, J. A., Reusch, T. B. H., Bonberg-Bauer, E., \& Olsen, J. L. (2016). Phylogeographic differentiation versus transcriptomic adaptation to warm temperatures in Zostera marina, a globally important seagrass.Molecular Ecology , 25, 5396-5411.

Kass, J. M., Anderson, R. P., Espinosa-Lucas, A., Juarez-Jaimes, V., Martinez-Salas, E., Botello, F., ... \& Sanchez-Cordero, V. (2020). Biotic predictors with phenological information improve range estimates for migrating monarch butterflies in Mexico. Ecography , 43, 341-352.

King, N. G., McKeown, N. J., Smale, D. A., \& Moore, P. J. (2018). The importance of phenotypic plasticity and local adaptation in driving intraspecific variability in thermal niches of marine macrophytes.Ecography , 41, 1469-1484.

Kramer-Schadt, S., Niedballa, J., Pilgrim, J. D., Schroder, B., Lindenborn, J., Reinfelder, V., .. \& \& Wilting, A. (2013). The importance of correcting for sampling bias in MaxEnt species distribution models. Diversity and Distributions , 19, 1366-1379.

Lacap, C. D. A., Vermaat, J. E., Rollon, R. N., \& Nacorda, H. M. (2002). Propagule dispersal of the SE Asian seagrasses Enhalus acoroides and Thalassia hemprichii . Marine Ecology Progress Series , 235, 75-80.

Lapointe, B. E., Tomasko, D. A., \& Matzie, W. R. (1994) Eutrophication and trophic state classification of seagrass communities in the Florida Keys. Bulletin of Marine Science , 54, 696-717.

Larkum, A. W. D., Pernice, M., Schliep, M., Davey, P., Szabo, M., Raven, J. A., Lichtenberg, M., Brodersen, K. E., \& Ralph, P. J. (2018). Photosynthesis and metabolism of seagrasses. In: Larkum AWD, Kendrick GA and Ralph PJ (ed) Seagrasses of Australia: structure, ecology and conservation. Springer, pp 315-342.

Lobo, J. M., Jimenez-Valverde, A., \& Real, R. (2008). AUC: a misleading measure of the performance of predictive distribution models. Global Ecology and Biogeography , 17, 145-151.

Lowe, W. H., \& Allendorf, F. W. (2010). What can genetics tell us about population connectivity? Molecular Ecology , 19, 3038-3051.

Lowen, J. B., Hart, D. R., Stanley, R. R., Lehnert, S. J., Bradbury, I. R., \& DiBacco, C. (2019). Assessing effects of genetic, environmental, and biotic gradients in species distribution modelling. ICES Journal of Marine Science, , 76, 1762-1775.

Lyimo, T. J., Mvungi, E. F., Lugomela, C., \& Bjork, M. (2006). Seagrass biomass and productivity in seaweed and non-seaweed farming areas in the east coast of Zanzibar, Tanzania. Western Indian Ocean Journal of Marine Science , 5, 141-152.

Lyimo, T. J., Mvungi, E. F., \& Mgaya, Y. D. (2008). Abundance and diversity of seagrass and macrofauna in the intertidal areas with and without seaweed farming activities on the east coast of Zanzibar. Tanzania Journal Science, 34, 42-52.

Mammola, S., \& Cardoso, P. (2020). Functional diversity metrics using kernel density $n$-dimensional hypervolumes. Methods in Ecology and Evolution , 11, 986-995.

Marin-Guirao, L., Ruiz, J. M., Dattolo, E., Garcia-Munoz, R., \& Procaccini, G. (2016). Physiological and molecular evidence of differential short-term heat tolerance in Mediterranean seagrasses.Scientific Reports , 
6,28615 .

Martins, A. R. O., \& Bandeira, S. O. (2001). Biomass distribution and leaf nutrient concentrations and resorption of Thalassia hemprichii at Inhaca Island, Mozambique. South African Journal of Botany , 67, $439-442$.

Mukai, H. (1993). Biogeography of the tropical seagrasses in the western Pacific. Australian Journal of Freshwater Research , 44, 1-17.

Muscarella, R., Galante, P. J., Soley-Guardia, M., Boria, R. A., Kass, J. M., Uriarte, M., \& Anderson, R. P. (2014). ENMeval: An R package for conducting spatially independent evaluations and estimating optimal model complexity for Maxent ecological niche models. Methods in Ecology and Evolution , 5, 1198-1205.

Melo-Merino, S. M., Reyes-Bonilla, H., \& Lira-Noriega, A. (2020). Ecological niche models and species distribution models in marine environments: A literature review and spatial analysis of evidence.Ecological Modelling , 415, 108837.

Merila, J., \& Hendry, A. P. (2014). Climate change, adaptation, and phenotypic plasticity: the problem and the evidence. Evolutionary Applications , 7, 1-14.

Miyoshi, K., Hattori, R. S., Strussmann, C. A., Yokota, M., \& Yamamoto, Y. (2020). Phenotypic/genotypic sex mismatches and temperature-dependent sex determination in a wild population of an Old World atherinid, the cobaltcap silverside Hypoatherina tsurugae. Molecular Ecology , 29, 2349-2358.

Olsen, Y. S., Collier, C., Ow, Y. X., \& Kendrick, G. A. (2018). Global warming and ocean acidification: effects on Australian seagrass ecosystems. In: Larkum AWD, Kendrick GA and Ralph PJ (ed) Seagrasses of Australia: structure, ecology and conservation. Springer, pp. 705-742.

Orth, R. J., Carruthers, T. J. B., Dennison, W. C., Duarte, C. M., Fourqurean, J. W., Heck, Jr. K. L., ... \& Williams, S. L. (2006). A global contemporary crisis for seagrass ecosystems. Bioscience, 56, 987-996.

Oney, B., Reineking, B., O’Neill, G., \& Kreyling, J. (2013). Intraspecific variation buffers projected climate change impacts on Pinus contorta. Ecology and Evolution , 3, 437-449.

Peterson, M. L., Doak, D. F., \& Morris, W. F. (2019). Incorporating local adaptation into forecasts of species' distribution and abundance under climate change. Global Change Biology , 25, 775-793.

Phillips, S. J. (2017). A Brief Tutorial on Maxent. Available from URL: http://biodiversityinformatics.amnh.org/open_source/maxent/. Accessed on 2021-02-10.

Phillips, S. J., Anderson, R. P., Dudik, M., Schapire, R. E., \& Blair, M. E. (2017). Opening the black box: An open-source release of Maxent.Ecography , 40, 887-893.

Pinsky, M. L., Selden, R. L., \& Kitchel, Z. J. (2020). Climate-driven shifts in marine species ranges: scaling from organisms to communities. Annual Review of Marine Science , 12, 153-179.

Radosavljevic, A., \& Anderson, R. P. (2014). Making better Maxent models of species distributions: complexity, overfitting and evaluation. Journal of Biogeography , 41, 629-643.

Ralph, P. J., CRosswell, J. R., Cannard, T., \& Steven, A. D. L. (2018). Estimating seagrass blue carbon and policy implications: the Australian perspective. In: Larkum AWD, Kendrick GA and Ralph PJ (ed) Seagrasses of Australia: structure, ecology and conservation. Springer, pp 743-758.

Razgour, O., Forester, B., Taggart, J. B., Bekaert, M., Juste, J., Ibanez, C., .. \& \&anel, S. (2019). Considering adaptive genetic variation in climate change vulnerability assessment reduces species range loss projections. Proceedings of the National Academy of Sciences USA , 116, 10418-10423.

Repolho, T., Duarte, B., Dionisio, G., Paula, J. R., Lopes, A. R., Rosa, I. C., .. \& Rosa, R. (2017). Seagrass ecophysiological performance under ocean warming and acidification. Scientific Reports , 7, 41443. 
Roberts, D. R., Bahn, V., Ciuti, S., Boyce, M. S., Elith, J., Guillera-Arroita, G., .. \& Dormann, C. F. (2017). Cross-validation strategies for data with temporal, spatial, hierarchical, or phylogenetic structure. Ecography , 40, 913-929.

Robinson, L. M., Elith, J., Hobday, A. J., Pearson, R. G., Kendall, B. E., Possingham, H. P., \& Richardson, A. J. (2011). Pushing the limits in marine species distribution modelling: lessons from the land present challenges and opportunities. Global Ecology and Biogeography, 20, 789-802.

Robinson, N. M., Nelson, W. A., Costello, M. J., Sutherland, J. E., \& Lundquist, C. J. (2017). A systematic review of marine-based species distribution models (SDMs) with recommendations for best practice.Frontiers in Marine Science , 4, 421.

Saunders, M. I., Leon, J., Phinn, S. R., Callaghan, D. P., O’Brien, K. R., Roelfsema, C. M., Lovelock, C. E., Lyons, M. B., \& Mumby, P. J. (2013). Coastal retreat and improved water quality mitigate losses of seagrass from sea level rise. Global Change Biology , 19:2569-2583

Sherman, C. D. H., Smith, T. M., York, P. H., Jarvis, J. C., Ruiz-Montoya, L. R., \& Kendrick, G. A. (2018). Reproductive, dispersal and recruitment strategies in Australian seagrasses. In: Larkum AWD, Kendrick GA and Ralph PJ (ed) Seagrasses of Australia: structure, ecology and conservation. Springer, pp 213-256.

Short, F.T., Carruthers, T., Dennison, W., \& Waycott M. (2007). Global seagrass distribution and diversity: A bioregional model. Journal of Experimental Marine Biology and Ecology , 350, 3-20.

Smale, D. A., Wernberg, T., Oliver, E. C. J., Thomen, M., Harvey, B. P., Straub, S. C., .. \& Moore, P. J. (2019). Marine heatwaves threaten global biodiversity and the provision of ecosystem services. Nature Climate Change , 9, 306-312.

Smith, A. B., Godsoe, W., Rodriguez-Sanchez, F., Wang, H. H., \& Warren, D. (2019). Niche estimation above and below the species level.Trends in Ecology \& Evolution, 34, 260-273.

Spalding, M. D., Fox, H. E., Allen, G. R., Davidson, N., Ferdana, Z. A., . \& \& Robertson, J. (2007) Marine ecoregions of the world: a bioregionalization of coastal and shelf areas. BioScience , 57, 573-583.

Thomson, J. A., Burkholder, D. A., Heithaus, M. R., Fourqurean, J. W., Fraser, M. W., .. \& Kendrick, G. A. (2015). Extreme temperatures, foundation species, and abrupt ecosystem change: an example from an iconic seagrass ecosystem. Global Change Biology , 21, 1463-1474.

Unsworth, R. K. F., McKenzie, L. J., Nordlund, L. M., \& Cullen-Unsworth, L. C. (2018). A changing climate for seagrass conservation? Current Biology , 28, R1221-R1232.

Valavi, R., Elith, J., Lahoz-Monfort, J. J., \& Guillera-Arroita, G. (2019). blockCV: An r package for generating spatially or environmentally separated folds for k-fold cross-validation of species distribution models. Methods in Ecology and Evolution, 10, 225-232.

Vale, C. G., Tarroso, P., \& Brito, J. C. (2014). Predicting species distribution at range margins: testing the effects of study area extent, resolution and threshold selection in the Sahara-Sahel transition zone.Diversity and Distributions , 20, 20-33.

Waycott, M., Duarte, C.M., Carruthers, T. J. B., Orth, R. J., Dennison, W. C., Olyarnik, S., .. \& \& Williams, S. L. (2009). Accelerating loss of seagrasses across the globe threatens coastal ecosystems. Proceedings of the National Academy of Sciences USA , 106, 12377-12381.

Wu, K. Y., Chen, C. N. N., \& Soong, K. (2016). Long distance dispersal potential of two seagrasses Thalassia hemprichii and Halophila ovalis . PLoS ONE , 11, e0156585.

Zhang, J. P., Huang, X. P., \& Jiang, Z. J. (2014) Physiological responses of the seagrass Thalassia hemprichii (Ehrenb.) Aschers as indicators of nutrient loading. Marine Pollution Bulletin , 83, 508-515. 
Zhang, Z., Capinha, C., Karger, D. N., Turon, X., MacIsaac, H. J., \& Zhan, A. (2020a). Impacts of climate change on geographical distributions of invasive ascidians. Marine Environmental Research , 104993.

Zhang, Z., Mammola, S., McLay, C. L., Capinha, C., \& Yokota, M. (2020b). To invade or not to invade? Exploring the niche-based processes underlying the failure of a biological invasion using the invasive Chinese mitten crab. Science of The Total Environment, 138815.

Zhang, Z., Mammola, S., Liang, Z., Capinha, C., Wei, Q., Wu, Y., .. \& \& Wang, C. (2020c). Future climate change will severely reduce habitat suitability of the Critically Endangered Chinese giant salamander. Freshwater Biology , 65, 971-980.

Zhang, Z., Kass, J. M., Mammola, S., Koizumi, I., Li, X., Tanaka, K., . . \& Usio, N. (2021). Lineage-level distribution models lead to more realistic climate change predictions for a threatened crayfish.Diversity and Distributions .

\section{Data availability}

Presence records of Thalassia hemprichii will be made publicly available in Dryad upon acceptance. Environmental layers can be downloaded from the Global Marine Environment Datasets (http://gmed.auckland.ac.nz) and the Bio-ORACLE database v2.1 (https://www.bio-oracle.org). Microsatellite data sets will be deposited in Dryad entry available soon.

\section{Author contributions}

Z.-M.H. and Z.Z. conceived the project. Z.-M.H. and J.Z. collected molecular data, J.Z. and P.F. performed genetic analyses, Z.Z. collected species distribution data and conducted ecological niche analyses with critical suggestions from J.M.K. and S.M. Z.-M.H., Z.Z., S.M., J.M.K., Z.-Q.Z., S.D., J.A., and A.J. interpreted and discussed the results, wrote and/or revised the manuscript. All authors approved the final version of the manuscript.

\section{Tables}

Table 1. Maxent parameters, performance metrics on spatially withheld data (validation AUC and omission rate) and the full dataset (continuous Boyce index), and 10th percentile presence thresholds of the two lineagelevel models and the species-level model. Validation AUC and 10\% omission rate results are expressed as means +- standard deviation across spatial partitions.

\begin{tabular}{|c|c|c|c|c|c|c|}
\hline Model & $\mathrm{RM}^{1}$ & Feature class ${ }^{2}$ & $\begin{array}{l}\text { Average } \\
\text { validation } \\
\text { AUC }\end{array}$ & $\begin{array}{l}\text { Average } 10 \% \\
\text { omission rate } \\
(\%)\end{array}$ & $\begin{array}{l}10 \% \text { omission } \\
\text { threshold }\end{array}$ & $\begin{array}{l}\text { Continuous } \\
\text { Boyce index }\end{array}$ \\
\hline $\mathrm{WTIP}^{3}$ & 0.5 & LQ & $0.99( \pm 0.01)$ & $3.57( \pm 7.14)$ & 0.12 & 0.92 \\
\hline $\mathrm{CTIP}^{4}$ & 2.5 & $\mathrm{LQH}$ & $0.96( \pm 0.03)$ & $\begin{array}{l}26.69( \pm \\
37.42)\end{array}$ & 0.37 & 0.99 \\
\hline Species & 0.5 & $\mathrm{H}$ & $0.96( \pm 0.02)$ & $\begin{array}{l}17.93( \pm \\
17.62)\end{array}$ & 0.30 & 0.99 \\
\hline
\end{tabular}

${ }^{1} \mathrm{RM}$ : regularization multiplier.

${ }^{2}$ Feature Class: L (linear), Q (quadratic), and H (hinge) were considered.

${ }^{3}$ WTIP: the Western Tropical Indo-Pacific lineage-level model 
${ }^{4}$ CTIP: the Central Tropical Indo-Pacific lineage-level model

Table 2 . Permutation importance (\%) of marine predictors in each Maxent model. Values in bold showed important predictors.

\begin{tabular}{llll}
\hline Predictors & WTIP $^{1}$ & $\mathrm{CTIP}^{2}$ & Species \\
Water depth $[\mathrm{m}]$ & $\mathbf{1 4 . 1 0}$ & $\mathbf{5 1 . 0 5}$ & $\mathbf{4 6 . 9 1}$ \\
Distance to land $[\mathrm{km}]$ & $\mathbf{8 1 . 8 7}$ & $\mathbf{1 1 . 5 8}$ & $\mathbf{9 . 3 0}$ \\
Minimum current velocity $[\mathrm{m} / \mathrm{s}]$ & 0.46 & 0.00 & 0.03 \\
Annual mean current velocity $[\mathrm{m} / \mathrm{s}]$ & 0.15 & 0.04 & 0.27 \\
Annual mean sea surface salinity $[\mathrm{PSS}]$ & 0.80 & 2.49 & 1.92 \\
Annual range of sea surface salinity $[\mathrm{PSS}]$ & 0.93 & 4.96 & $\mathbf{1 3 . 3 8}$ \\
Annual mean sea surface temperature $\left[{ }^{\circ} \mathrm{C}\right]$ & 0.83 & $\mathbf{2 8 . 6 8}$ & $\mathbf{2 3 . 4 7}$ \\
Annual range of sea surface temperature $\left[{ }^{\circ} \mathrm{C}\right]$ & 0.87 & 1.20 & 4.73 \\
\hline
\end{tabular}

${ }^{1}$ WTIP: Western Tropical Indo-Pacific lineage-level model

${ }^{2}$ CTIP: Central Tropical Indo-Pacific lineage-level model

Table 3. Range size change (\%) of Thalassia hemprichiiunder future climate scenarios. Values in parentheses indicate range size change predicted by the species-level model.

\begin{tabular}{lllll}
\hline RCP $^{1}$ & WTIP $^{2}$ & WTIP $^{2}$ & CTIP $^{3}$ & CTIP $^{3}$ \\
& $2050 \mathrm{~s}$ & $2100 \mathrm{~s}$ & $2050 \mathrm{~s}$ & $2100 \mathrm{~s}$ \\
RCP 2.6 & $-0.2(-2.5)$ & $4.4(-3.8)$ & $-40.6(-29.3)$ & $-34.5(-27.8)$ \\
RCP 4.5 & $1.3(-6.3)$ & $4.2(-6.3)$ & $-49.7(-26.1)$ & $-55.7(-26.4)$ \\
RCP 6.0 & $3.4(-0.4)$ & $7.5(-15.0)$ & $-43.8(-30.3)$ & $-63.7(-23.2)$ \\
RCP 8.5 & $4.0(-10.9)$ & $13.2(-23.7)$ & $-53.7(-27.4)$ & $-72.1(-25.8)$ \\
\hline
\end{tabular}

${ }^{1}$ RCP: representative concentration pathway.

${ }^{2}$ WTIP: Western Tropical Indo-Pacific lineage-level model

${ }^{3}$ CTIP: Central Tropical Indo-Pacific lineage-level model

\section{Figure Legends}

Figure 1. (a) Map of study regions and presence records used in this study. Red and blue points represent sample collection locations for molecular analyses in the Western Tropical Indo-Pacific (WTIP) and the Central Tropical Indo-Pacific (CTIP). Gray crosses show presence records used for the species distribution models. (b) Network of microsatellite genetic differentiation (Cavalli-Sforza and Edwards chord distances). The topology results from pruning the network for pairwise genetic distances $<0.534$. The smallest chord distance (0.499) between the WTIP and CTIP lineages is shown. (c) Genetic lineage division over space based on STRUCTURE clustering $(\mathrm{k}=2)$. Population abbreviations are the same as in Hernawan et al. (2017) and Jahnke et al. (2019a), and their classification to network analysis is in agreement with that of STRUCTURE (Fig. 1c).Figure 2. The realized niches for the two lineages of Thalassia hemprichii quantified via eight-dimensional hypervolumes. Circles with white rims indicate hypervolume centroids. Boundaries and shapes of hypervolumes were delineated by 10,000 points randomly sampled within each hypervolume. The photograph depicts T. hemprichii.Figure 3. Present-day continuous (a, b) and binary $(\mathrm{c}, \mathrm{d})$ habitat suitability predictions for Thalassia hemprichii by lineage-level $(\mathrm{a}, \mathrm{c})$ and species-level $(\mathrm{b}, \mathrm{d})$ Maxent models. Dashed lines represent the equator. 
Figure 4 . Changes in continuous (a, b) and binary (c, d) habitat suitability for Thalassia hemprichii projected by lineage-level (a, c) and species-level (b, d) Maxent models under the RCP 8.5 scenario in the 2050s. Dashed lines indicate the equator. The category "stable" represents areas predicted to be suitable under both present-day and future climatic conditions, "loss" indicates areas predicted to be suitable under present-day conditions but unsuitable in the future, and "gain" indicates areas predicted to be unsuitable under present-day conditions but suitable in the future.

\section{Supporting Information}

Additional supporting information can be found online in the Supporting Information section at the end of the article.

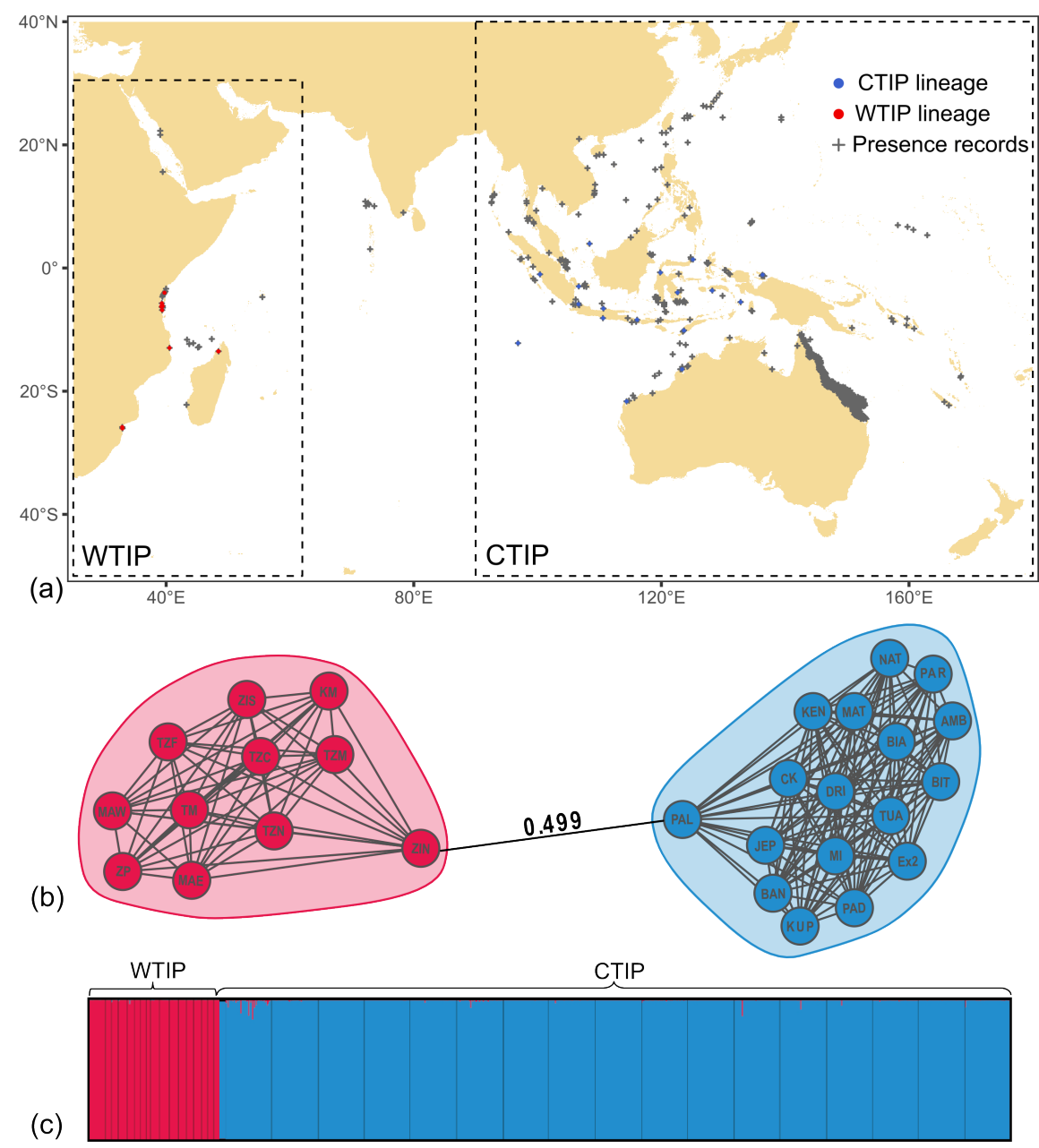




\section{Hosted file}

Figure 2.pdf available at https://authorea.com/users/342403/articles/511956-intraspecificgenetic-variation-matters-when-predicting-seagrass-distribution-under-climate-change
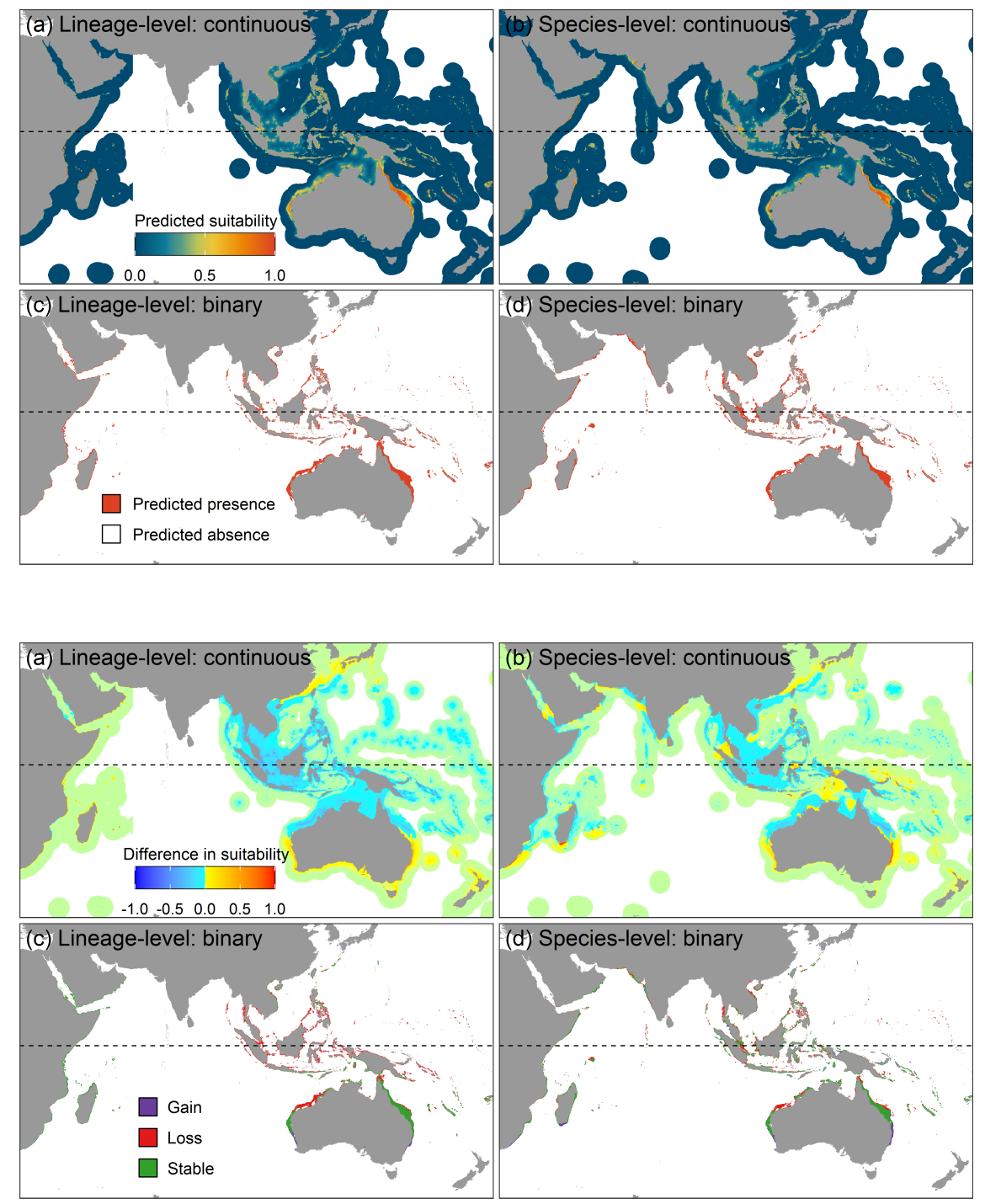